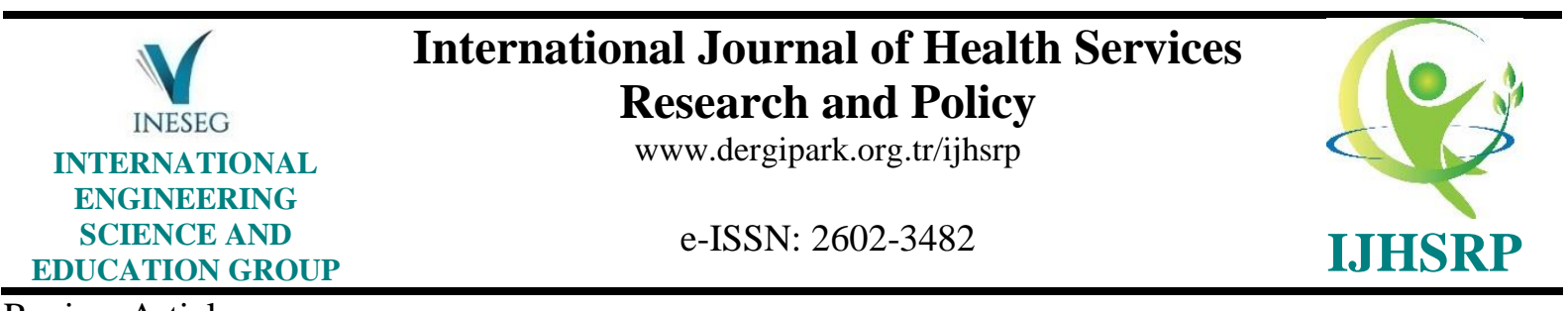

Review Article

\title{
TOWARD ORGANIZATIONAL EVIDENCE-BASED MANAGEMENT IN HEALTHCARE ORGANIZATIONS
}

\author{
Hatem H. Alsaqqa*:0
}

Ph.D. Department of Health Management, Faculty of Health Sciences, Ankara University/TURKEY

* Corresponding author: hs-mch@ @hotmail.com

\begin{abstract}
To date, relatively little evidence has been published as to what represents effective evidencebased management in healthcare organizations. Existing studies are rarely conceived or developed with respect to organizational factors determining whether such studies succeed or fail. One of the challenges in linking organizational factors to evidence-based management is to identify the focus at which characteristics of healthcare organizations and management of knowledge resources are most obvious. This paper sets out to sense this issue. The paper suggests a conceptual framework through reviewing the existing literature on organizational factors and evidence-based management in the healthcare sector. A new conceptual approach as to how organizational factors and managerial-decision process can be more effectively linked through the organizational-level of healthcare organizations. Recommendations are provided with regard to how future healthcare organizations can approach the evidence-based management from a logically wide organizational-level and context perspective. The present paper represents an attempt to link organizational factors and evidence-based management in a more meaningful way. A conceptual model has been provided as a way to frame and imagine the organizational circle of producing management evidence.
\end{abstract}

Keywords: Context; Organizational characteristics; Organizational culture; Evidence-based management; Healthcare organizations

\begin{tabular}{ll}
\hline Received: February 12, 2020 & Accepted: June 2, 2020 \\
\hline
\end{tabular}

\section{Introduction}

Healthcare organizations (HCOs) today confronting so much information and knowledge processing, as huge data, which are produced daily by healthcare providing process. Managers have time and resources constraints that restrict the degree to which they can participate in evidence-based management and often lack the capabilities needed for evidence-based management, formulate organizational management issues, access colloquial and level data, investigate the evidence and apply it to decision-making. That lead to arguments about the need for standardization of knowledge and practice within HCOs.

Significant efforts in the conception of HCOs, motivated by improving the HCOs performance and focusing on accountability for quality, costs, and other measures. Not only the clinical decisions on 
each patient's care must be improved to ensure healthcare is safe and efficient, but also the managing choices on how to manage it. It encourages managers to popularize their management perspectives and achieving robust sources of evidence for their decision-making. Healthcare Information and knowledge advances are opportunities that can facilitate the implementation of an evidence approach for HCOs management. If, however, this expertise is channeled into the evidence-based management (EBMgt) remit, a Trojan horse may seem to foster a 'top-down' hierarchy of organizational evidence. One way of finding out the most important sophisticated knowledge for HCOs with professionals is cooperating at different levels by organizing and disseminating.

Research on HCOs decision-making in the United States, Canada, and the UK suggests that the extent to which a decision-making procedure is used varies considerably and that managers seldom take advantage of evidence when making decision-making [1,2]. This unacceptable state of affairs is due to two main reasons. The first is that researchers have been more focused on testing theory and its explanation, rather than producing managerial interventions to better corporate managers practice. The other is that managers do not consider evidence rather than perspectives to cope with evidence.

It is shown that healthcare administrators have begun to accept the practice of EBMgt. Influenced by the evidence-based medicine or evidence-based practice movement, more and more healthcare administrators understand that when taking managerial decisions, the evidence-based approach must be taken [3]. Decisions based exclusively on personal experience and intuition are not prudent. Decisions have to be made in addition to the experience of HCOs' administrators on the basis of the best available evidence, organizational details, stakeholder concerns, and principles [3].

Tranfield et al. [4] recognize this need and compare research on management with medical research, in other words on dimensions including discipline nature, research culture, the design of research, the review protocol, and so on. They argue that the nature of management diverges while medicine converges. As a result, research culture on medicine is rigorously assessed, while management research is divided between the positive and phenomenological perspectives [4]. Rousseau and McCarthy [5] point to the improvement of managerial decision-minding and organizations achieving results when management education is focused on evidence [6].

Regarding the use of EBMgt, researchers have attempted to explain the difficulties that EBMgt can have for managers. The fact that managers and executives could not use enough evidence and the existing evidence does not fully apply was one of the apparent reasons [7]. As managers face responsibilities for the performance of their organizations, Bigelow and Arndt [8] wanted to know if something in the research helps them to better manage their organizations. Some scholars have acknowledged that a restricted management research base [9] and a gap between healthcare research and practice [9] have existed. Following the EBMgt debate, scholars believe that management evidence should be seen in a wide context [2].

\subsection{Definition, meaning, and significance of evidence-based management}

In the last 20 years, an intensive effort has been made to expand new organizational and governance frameworks and models, particularly in the healthcare sector [10]. Evidence-based management is defined as making decisions about the management of employees, teams, or organizations through the conscientious, explicit, and judicious use of the best available scientific evidence, organizational data, professional experiential evidence, and stakeholders' values and concerns [11]. Evidence-based originally derived from medical sciences in the 1990s, today's doctrines develop 
in disciplines such as education, nursing, criminology, social sciences. But EBMgt is still in infancy in HCOs management $[12,13]$. Important articles and strongly recommended EBMgt practice in the management of healthcare [8]. This "evidence" could be derived from research, internal organizational information, and even administrative experience [10].

Information from healthcare stakeholders, scientists, and professionals are required to fill gaps in this regard. This strategy of evidence-based decisions was clarified by Bongers [14] as trans-disciplinary where discipline integration is regarded as central and requires critical thinking and reflection, and the application of the best possible evidence. Evidence in EBMgt supports a tool to answer questions about the expected outcome of a decision. Hospital managers are therefore obliged to use the EBMgt to achieve efficient administration. EBMgt can improve the quality of organizational decisions and have a decisive impact on performance as a bridge from theory to practice [15].

Healthcare administrators are health officials whose decisions have a major impact on the efficiency of quality patient care and HCOs' success. Safety of patients, quality of care, and access and widespread demands for a reduction in care costs and value for money all demand an evidence-informed approach from healthcare administrators in decision-making [16]. An EBMgt approach to their management and administration is essential for healthcare administrators [16].

This multidisciplinary approach exposes HCOs to a series of expertise and evidence which can have a positive impact on decision-making within healthcare systems by the senior managers. For Rousseau, social science and organizational research should inform such decisions to decrease reliance on personal experiences rather than systematic knowledge [17]. In its management and organizational research, EBMgt recommends the use of the decent scientific practice of systematic syntheses. Such guidance requires efficient and competent management of the provision of healthcare services either at an institutional level or at an organization or macro level. Several experts have pointed to the significance of synthesis in assessing knowledge claims, quality control, threats to integrity, and preventing knowledge loss $[18,19]$. It is, therefore, possible to come to the conclusion that EBMgt practices represent essentially a combination of critical thinking and evidence from various sources in order to promote the probability of positive results [20].

Axelsson [21] argued that healthcare managers should learn to think and appraise evidence from management research as a basis for their works. There are many viewpoints regarding EBMgt that are inspired and presented by organizations' specialists [18]. The administrators of HCOs have to learn the principles and processes of EBMgt, develop the abilities to search for information, assess the quality of evidence, and apply the evidence for their healthcare decision-making to ensure that the quality of healthcare and the cost of care is reduced. Providing EBMgt training would increase the knowledge of EBMgt and decision-making skills in HCOs [4].

EBMgt literature criticizes a corporate and administrative approach, which lacks evidence-based research in healthcare systems and organizations. The reasons provided are the pending danger of overuse, abuse, and underuse of information that can, in the end, have consequences for the well-being and efficient management of organizations [8, 18]. It is in the literature for a long time that management knowledge can and should become practical, so practices can benefit [17]. However, for a number of reasons it has not yet come to the heart of the practice, practitioners always rely on their own judgment or traditional convictions. Many researchers inspected the reasons for this unfortunate disconnection between management research and practice [15]. 
EBMgt is a way to regulate the methods of collecting, evaluating, and producing better standards and guidance. It is a method to manage the market of management ideas, which are regulated, controlled, evaluated, and therefore deemed more reliable in terms of the high quality of business knowledge [7]. So, if little or no reliable information is available for decision-making, managers are trying to do something based on evidence-based managing methods rather than guessing and expecting [7] on the basis of logic and evidence. Another valid argument is that evidence-based management is affected by the disease itself that it seeks to cure. In other words, while evidentiary management is attempting to promote the adoption of a cumulative body of management knowledge that managers validate, verify, and use, it does not boast a strong body of know-how. The lack of a hypothetical framework in the field could be one reason for this [22].

In case of that, the most relevant definition for our purposes of evidence is the factual, systematized form of information or observation presented to support or justify beliefs or inferences [23, 24]. For evidence to be useful, it must be rigorous and relevant to the context in which it is invoked. In other words, rigor and relevance are at the core of evidence generation and evaluation [15]. Pfeffer and Sutton [7] suggested that management can learn from the successful steps that other occupations, including medicine, have taken towards evidence-based practice in order to become EBMgt in a real organizational environment.

\section{Methodology/approach}

This is a conceptual framework that incorporates various types of viewpoints linked to each other. The framework includes coordination in various divisions of the organizations, based on research in the context of healthcare. This cooperation can also be primarily coordinated by multidisciplinary teams across the boundaries of different healthcare organizations.

The concept model is designed to incorporate evidence-based concepts to the literature in the management of healthcare organizations. The conceptual structure involves organizational tasks that must be undertaken in separate units by professional personnel. It aims to provide a fairly precise definition of entry-level decision making, better management of knowledge management resources (information, experience, research) in various stages, and an overview of broader dimensions (context, organizational features, corporate culture). The model is complemented by a series of efficient circles to assess organizational awareness, relevant findings, and recurrent awareness use and create a clear database for decision-making. It will provide sufficient details to test the concept in practice.

Searches were conducted of the commonly used health databases to identify relevant literature. Major health electronic databases including Emerald, EBSCO, Sage, PubMed, and Taylor and Francis were searched to retrieve appropriate published articles that may be applicable to research objectives. Three sets of search terms were used, namely, (1) 'evidence-based' and (2) 'healthcare organization' and (3) 'management'. Additional references were identified through examination of the references from most relevant publications (snowballing).

Inclusion criteria were: (1) journal articles and grey literature written in English spanning the previous years; (2) studies that include evidence-based in healthcare organizations management. Exclusion criteria were: (1) studies that focused on clinical evidence or medicine evidence and (2) studies did not consider the evidence-based concept either they are related to healthcare organizations' management. 
The researcher checked the titles and abstracts of papers for significance, and where needed, the full text of papers was retrieved. For their contribution to the interpretation, literature, and philosophical understanding of evidence-based management, domain identifications, and alternative models, full-text papers were analyzed. Firstly, evidence that linked to the concept and definition of evidence-based management was identified. Secondly, evidence of health-care organizations has recently been considered and further work is still required to be formally adopted.

\subsection{Conceptual model of evidence-based management}

From the previous definitions of EBMgt, the author conceptualizes a model that may help when evidence has to be considered in the management of HCOs. The author considered three strategic criteria and three dissimilar processes to accomplish his model (Figure 1.). In addition, the author accepted the evidence to be at the organizational-level of HCOs, in case of evidence at this level may be more obvious and applicable to use the concept. The three strategic criteria included the organization's context, characteristics, and culture. The three processes included the organizational knowledge process, the knowledge usage and outcome cycle, and also the repeating process which makes the second process to be evidence for the same organization itself and other organizations. However, this model is an attempt to make the handling of evidence in management more rational and logical for managers, but not to be a guarantee as a standard for every managerial procedure or decision-making phase. The aim of this conceptual extraction model was to highly lighten and enhance the role of evidence mindset in the domains and evaluations of management for more rational and timely successful healthcare decisions, which can be a basis for future evaluation.

\subsection{Context, characteristics, and culture of healthcare organizations}

In the healthcare industry, there are many intangible properties and intellectual properties. It has many distinct characteristics besides being intensive knowledge than other sectors. The variables that influence Knowledge management (KM) can be organizational features, expert opinions, and planning in compliance with the literary assessment [25]. Many studies have discovered that more resources and instruments are being used as the scale (size) of the organization increases to introduce fresh information technology [25]. Various dimensional HCOs have a distinct attitude to the handling and use of the healthcare industry information.

Organizational culture is a summary of all members' particular values and organizational laws that affect communication between external parties and members of the organization. Tolfo and Wazlawick [26] proposed that members should share the organizational culture's values, beliefs, and laws. Wallach [27] split the organizational culture into the culture of bureaucracy, creative culture, and support culture. Four kinds of competing values have been suggested in Quinn [28]: rational culture, hierarchical culture, group culture, and growth culture. The features of DeshPandé and Farley [29] more or less demonstrate the rational culture, hierarchical culture, collective culture, and growth culture, as staff defines the culture of the workplace. However, one of these types usually dominates.

However, some studies indicate that when significant HCOs confronted issues, executives did not first consult with informed results. If EBMgt is not connected to efficient organizational environments, it is unlikely to achieve an extremely truthful choice on the quality of care. Although culture is described as a significant variable by Eddama and Coast [30] affecting the extent to which "reasonable" healthcare investment choices are based on evidence. A comprehensive study has been carried out on the prevalent 
values in HCOs, and while the literature on the connection between culture and accomplishment is increasing, there is a slight focus on decision-making either as an endpoint or as a flexible interference.

In reality, culture has been defined as the hardest organizational concept to define, making it hard to assess the effect on decision-making. Clearly, culture is the decision-making method that can be inferred, but there is a need for proof to demonstrate how this occurs. The literature highlights the significance of study culture as a precondition for policymaking based on evidence. Hyder et al. [31] disclosed in their research of low and middle-income nations that the informant recognizes this statement. Given the present organizational and administrative culture of the healthcare system, if important capability weaknesses of the service feature as constraints, it would involve important changes in the activities of healthcare systems [32].

Arndt and Bigelow [7] asserted that their professionals could generalize outcomes across organizations and systems, promoting an EBMgt as a feasible decision-making model. Arndt and Bigelow indicated that EBMgt reflected the assumptions of evidence-based medicine in relation to the systematic implementation of the finest evidence available to assess organizational policies. Managers should review the results of appropriate studies and practice syntheses regularly before making significant choices [32]. While there is no powerful evidence and a systematic study suggesting that evidence-based management actually increases organizational efficiency and promotes managers' decision-making [33]. The generation of evidence can persuade the public to make better decisions within the organization through an evidence-based management approach [15].

Organizations face fresh dynamics of dissimilar cultures and situations in their settings, and managers are needed to create the correct choices through evidence from other cultures and environments. This requires more comparative studies seeking emerging universality and cultural specificity at the same time [34]. This problem shows the significance of including contextual variables as a significant source of conflicting results in the literature to enhance convergence and acknowledge contextual differences [35].

Contextualization attention has risen considerably over the past 30 years [36, 37]. The word context is used to refer to a business that is the appropriate setting for the practical application of study evidence. For reference to a community, the term context is used. These contextual factors are suggested to promote or limit the process of evidence-based decision-making at greater levels of evaluation. The gap between existing evidence and practice in healthcare management reinforces the necessity for more active resolves, without ignoring the organizational-level context. A greater understanding of this level can be significant in closing the undesired gap.

The structures and processes of each healthcare environment represent a unique organizational milieu, including the way an organization operates and acts. These structures (e.g., size, staffing) and processes (e.g. practical arrangements, decision support) are, individually or together, capable of promoting or hindering discreet action in terms of the adoption and application arrangements.

Our knowledge of what works is becoming increasingly essential to the organizational structure, not how it operates in implementing research structures and procedures in operational situations [38, 39]. However, as these activities are carried out in more organizations in diverse settings and places, their capacity to perform them in the way they were initially described and demonstrated to be efficient will continue to decrease without better and more specific inclusion of an organizational research framework into study implementation agendas [40]. 


\subsection{Organizational information resources and decision-making}

Healthcare administrators have indicated that organizational information in healthcare management has often been used for decision-making. The first type of information resource was organizational data [13]. The literature provides various levels of evidence used by managers and other healthcare professionals in their decision-making method. Evidence sources include; best accessible scientific research, organizational information, knowledge and judgment, and values and concerns of stakeholders [41, 42].

Management based on evidence is based on two assumptions. First, it is a linear decision-making method. For instance, Kovner \& Rundall [43] sets out a decision method to sequentially define issues, search for options, evaluate alternative solutions, choose options, and execute these solutions. Second, it is possible to generalize and replicate the outcomes of these choices across organizations. The degree to which an organization can identify and process fresh information is probable to affect levels of rationality (i.e. instrumentalism) in decision-making. However, as mentioned above, this understanding is not restricted to formal evidence. It promotes the significance of tacit knowledge for organizational memory, and hence the background to decision-making [2]. Healthcare executives classified data generated within the organization as the most important and most frequently used type of evidence [2].

Kovner and Rundall observed that big HCOs are predisposed to depend on external management consultants for their strategic decision-making and execution plans and that the data on which such suggestions are based is not rigorously challenged by healthcare managers. An EBMgt strategy will risk promoting the dissemination of standardized information in HCOs. The analysis also points out that decision-making must have a well-understood organizational culture and strategic orientation.

\subsection{Organizational evidence-based management}

EBMgt imports making choices by using the best accessible evidence from various sources accurately, clearly, and sensibly to improve the probability of a suitable result $[10,43]$. This approach is guided by the premise that the management evidence-practice gap is due not only to knowledge transfer issues but also to information manufacturing issues [44]. Managers, therefore, have a duty to make actual and effective choices that assist their organization's mission and vision, comparable to doctors who use the finest scientific evidence available in clinical patient choices [18]. However, between this perfect situation and the status quo [7, 45], there is a notable gap. From various research, the main factors were recognized. Several variables have influenced EBMgt, including organizational variables, facilitators, obstacles, policies, and individual and social variables [42].

Management based on evidence that translates best evidence-based values into organizational procedures. Practicing managers grow into specialists through evidence-based management that makes organizational choices informed by social science and organizational research. That leads to shifting professional choices away from personal preference and disorganized experience towards those based on the finest evidence presented $[18,46]$.

To bridge the gap between understanding of leadership and practice of leadership, we are encouraged to address the research-practice gap by the values of evidence-based management (EBMgt) [47]. Evidence can be a structured set of data submitted to support or justify one's views or inferences about a phenomenon of concern [23, 24]. Evidence can be derived from organizational understanding, insight, judgment, and experience of executives, distinctive environmental and organizational features, preferences of different stakeholders, external environment, etc [6, 47]. 
Common sense and empirical study support the concept that high-quality information leads to better choices [48]. Managers also understand the importance of such high-quality information, which is examined by a professional body that raises their professional status [49]. However, the selection of information sources by decision-makers is often determined by availability and not quality. In relation to accessibility, confidence is asserted to play a major part in the selection of information sources by decision-makers [50]. The notion of an autonomous organization accountable for assessing evidence and synthesizing results can assist narrow the gap between studies and practice.

Management based on evidence is conceptualized by interplaying evidence and context with a facilitating process organization [51]. Once knowledge is generated and enforced, feedback offers data that can identify obstacles to the application, contextual backgrounds, or unpredicted causal relationships. Organizational decision-making and execution of evidence are restricted by legal and ethical frameworks under which organizations operate [7]. The context in which knowledge is produced may differ in terms of politics, culture, economy, and so on from the context in which it is applied.

Practitioner managers are closer to day-to-day organizational issues and decision-making and are interested in gaining an actionable understanding that would allow them to create more efficient choices. Other main players are the disseminators of information, who are interested in information that can attract the greatest crowd and are therefore interested in significance. The objectives of each can be accomplished through close cooperation between these stakeholders. A structure that brings together rigor, significance, and actionability adds value by aligning the various interests and purposes. It is this alignment that offers the means and rationale for scientists, information disseminators, and practitioners' involvement and collaboration [15]. Kulik [52] claims that diversity management study is not helpful to professionals as it focuses more on how staff perceives appropriate practices than on whether such practices are efficient in attaining interesting organizational outcomes [15].

The EBMgt cooperation requires to define the characteristics of evidence to promote its application in order to produce practically helpful understanding. We contend that the synthesis of evidence should result in appropriate, thorough, and actionable information [53]. This range of sources well reflects the range of choices and judgments that healthcare practitioners (policymakers, organizational executives, and healthcare professionals) need to create on a daily basis [54] requiring distinct information and evidence levels.

Pfeffer and Sutton [7] recommend further that one of the explanations why managers do not make use of evidence as the basis for making decisions is that, it changes the dynamic authority of the organization. The decision power, as a key resource for decision-making rather than for organizational politics and structural powers, would be distributed according to the competence and master of evidence in a culture supported by evidence-based decision-making.

\subsection{Organizational knowledge management}

KM has lately become very important to health authorities in healthcare organizations. It is prepared to acquire the expertise to guarantee more effective information management, to enable healthcare employees to work in a virtual environment, and to enhance resource efficiency [55]. Hongsermeier, et al. [56] say that the application of a KM system plays a vital part in enabling the flow of knowledge through its life cycle. It should be understood that KM will have credible, real-time information on healthcare officials to support decision-making for better outcomes. There are three components to the transfer of knowledge: people, processes, and technology. People of these three parts 
are the most significant pillar for information sharing. Therefore, adopting a healthy approach to the application of KM healthcare is incontrovertible [57, 58].

However, implementing and even succeeding KM programs within organizations is not a peaceful method. Their presence and strength are affected by elements such as organizational nature, type of organization, and service category. Given the sparse empirical information on how these variables affect $\mathrm{KM}$, this supports the requirement and importance of studying such variables in particular situations [6].

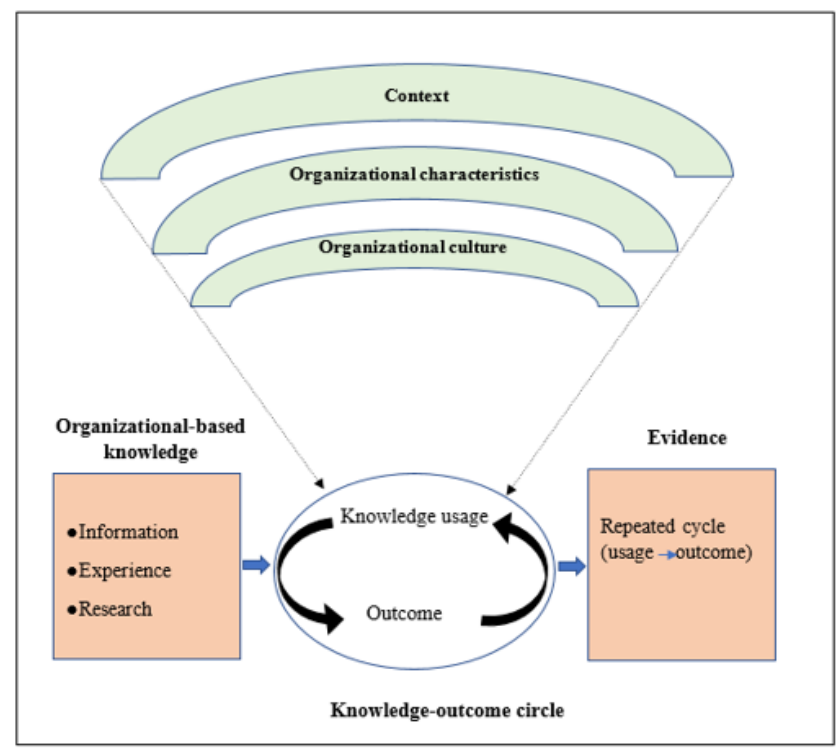

Figure 1. Conceptual framework of Organizational Evidence-based Management (developed by the author)

Scientific knowledge is needed for competent decision-making, but science cannot be only adequate to create choices [59]. Healthcare management is more probable to operate in a bad validity setting, like other management in general [60].

Implementation of KM system involves the use, transfer, and translation of information, prevention against a possible loss of understanding induced by retirement and employee turnover, acquiring competitive benefits, continuing learning, preventing organizational isolation and meeting user requirements [61]. Using knowledge means transferring it from rudimentary discovery to technical efficiency and then to acceptability processes, which indicates that this KM component involves two stages. This implies that knowledge is used by understanding, such as evidence-based rules. The third significant element of KM, the transfer of information, is the dissemination of information that is guided and regulated through distinct methods [62, 63].

The conversion of one type of understanding into the other and the group collaboration in the model system is essential. Knowledge database and management systems can be used to transform tacit knowledge into explicit knowledge. Tools such as decision support systems and electronic performance support systems are used to transform explicit knowledge into tacit knowledge [61]. KM is, therefore, an important factor for cooperation and information sharing in order to achieve an ideal outcome for the healthcare service [64]. To that end, the KM should be created accessible and easily accessible to anyone who wants it. Overall, the application of KM in the healthcare industry provides countless advantages. 
However, the gratitude of EBMgt as social technology needs you to regard variables such as culture and values as involved components of the social setting that encompass the formulation, practice, and reception of choices. Such factors should not be isolated and reflectively interpreted by other sources of evidence. Healthcare managers should be conscious of the change of projects and understandings and participate in critical, open, and questioning dialog in their social realities [65].

Organizational epistemology indicates that there are three characteristics that define organizational understanding more exactly [66]. First, the organizational view of multiple knowledge allows the organization to understand [67, 68]. Objective or personal information [67, 69] measurement for that reason is inappropriate. Measuring organizational understanding without acknowledging and assessing the characteristics of individuals who want to provide understanding is also difficult. Second, organizational knowledge scope and context [66]. Knowledge within organizations may be very general or very special. Third, organizational knowledge is captured through language. People use language to differentiate relevant knowledge from what should be ignored [66]. For example, Arndt and Bigelow [8] examine the evidence in the context of healthcare by noting that "best evidence" is an artifact of social processes resulting in its development that reflects the interests of researchers or organizations, which questions they have to ask and which sources of information they legitimize [60].

The use of study expertise is now receiving significant attention from EBMgt and policymaking through the HCOs. Another element to consider is the knowledge translation method, which includes operations such as synthesis, dissemination, and implementation of information. This is seen from the point of view of decision-makers or policymakers because their critical objective is to promote the integration of study information into program and policy development decisions [70]. Chen, Liu and Hwang [25] observed that a significant number of healthcare scientists have submitted applications and frameworks for KM, while few have studied factors for its implementing programs. The literature contains a wide variety of variables that are critical to effectively performed KM [71, 72].

\section{Discussion}

HCOs are multifaceted organizations due to their multiple and ambiguous objectives, the nature of their activities, and the diversity of professionals that perform their tasks by contributing to the provided services. In healthcare, decisions and actions result from political, interpretive, and symbolic aspects more than from essentially rationalistic approaches. As highly pluralistic contexts, organizational knowledge, legitimacy, and social capital play an important strategic role. However, their accumulations involve a variety of internal and external stakeholders concerned with both internal conditions and external factors. This context challenges the managers to find the balance between the use of rational managerial models, which balance social mission and sustainability with a set of institutional forces.

Kovner and other scholars stated that EBMgt would expand the capability of decision-makers and their inspiration to use more systematic methods in healthcare management decision-making [7, 43]. However, rare studies have been published to examine whether healthcare managers use an evidencebased method for running decision-making. Managers in healthcare prerequisites to increase information and knowledge to generate stronger evidence related to the impact of EBMgt on organizational performance. 
The EBMgt implementation process is influenced by the organizational culture, leadership, networks, resources, and evaluation, and feedback actions within HCOs. The concept of an independent organization responsible for evaluating evidence and synthesizing findings to increase its practical usefulness. Actually, we live in an era of "evidence-based everything", and that everything, medicine, management, economics, healthcare policy, and the other sciences-have become evidence-based [42, 73].

Despite its claims to rationality, EBMgt is actually presented in terms that evoke institutional theory. If a research study were to associate an outcome with a specific decision in a particular HCO, the use of EBMgt would have it serve as a basis for other organizations to solve a like problem and diffuse the practice in question. Rousseau [17] even suggests that the use of EBMgt produces legitimacy in the judgments of stakeholders. Such arguments reflect an institutional view of HCOs that focuses on the dissemination of practices that are approved by model organizations in order to sustain organizational legitimacy [74]. In that case, EBMgt merely creates new constraints that would add to the difficulties of HCOs to cope with internal complexity, sustain the adaptability needed to survive in a complex environment, and view unanticipated outcomes as inevitable or a "gift from an uncertain world" [75].

\section{Conclusions and future implications}

It is significant to shift proficient choices away from personal favorite and unsystematic knowledge towards those based on the finest organizational evidence available. An autonomous organization accountable for assessing evidence and synthesizing results can increase flexibility in the workplace information collaborations and presents guides for professionals to use. However, little is known about the diffusion of organizational EBMgt, but the assumption is needed to be widely disseminated.

The applicability of EBMgt within different HCOs urged to participate and cooperate more, in order to increase the amount and process of fresh information available in the field. Organizationalbased data, experience, and research networks should be expanded and adding to the EBMgt knowledgebase. HCOs in different sectors need to bring researchers, managers, and practitioners together to conduct more organizational EBMgt by collecting and organizing EBMgt information resources and provide the synthesized information for practicing. This exertion can help healthcare managers to consult the best available evidence for their decision-making.

After examining the nature of evidence as defined by advocates of EBMgt, the author close with a discussion of consequences and implications for HCOs management. The variety of how healthcare organizational aspects are outlined and defined complicates their measurements and the ability to integrate them into the decision-making progression. How individual and organizational constructs are intellectualized and measured in relation to the implementation process depend on (1) The conceptual model and organizational theory underlying the management level; (2) The nature of the organizational characteristics; (3) The size and the complexity of the organization itself.

Nevertheless, management based on evidence translates best evidence-based values into organizational procedures. For strategic or operational decisions, other kinds of information are useful but not sufficient. As a result, various sources of information must be considered by simple inspection of data trends and patterns. It is essential for managers to make informed decisions and to identify the 
relevance to the specific problem of evidence that appropriate management of knowledge. Likewise, this can help narrow the gap between management research and practice. For healthcare to be effective, timely, and reliable, the management and policy decisions have to be evidenced about how to organize, manage, and improve that care.

Ethics declaration: As my manuscript is a conceptual one, so it does not contain material and analysis of data. It also has not had an ethics committee's permission like a research article.

The compliance to the Research and Publication Ethics: This study was carried out in accordance with the rules of research and publication ethics.

\section{References}

[1] Arndt, M., Bigelow, B., "Evidence-Based Management in Health Care Organizations: A Critique of its Assumptions", In Academy of Management Proceedings, Vol. 1, pp.1-6, 2007.

[2] Guo, R., Berkshire, SD., Fulton, LV., Hermanson, PM., "Use of evidence-based management in healthcare administration decision-making”, Leadership in Health Services, 30(3), 330-42, 2017.

[3] Guo, R., Hermanson, PM., Farnsworth, TJ., "Study on Hospital Administrators' Beliefs and Attitudes toward the Practice of Evidence-Based Management", Hospital topics, 94(3-4), 62-6, 2016.

[4] Tranfield, D., Denyer, D., Smart, P., "Towards a methodology for developing evidence-informed management knowledge by means of a systematic review", British Journal of Management, 14(3), 207-22, 2003.

[5] Rousseau, DM., McCarthy, S., "Educating managers from an evidence-based perspective", Academy of Management Learning \& Education, 6(1), 84-101, 2007.

[6] Baba, VV., Hakem Zadeh, F., "Toward a theory of evidence-based decision making", Management decision, 50(5), 832-67, 2012.

[7] Pfeffer J, Sutton RI, "Evidence-based management”, Harvard business review, 84(1), 62, 2006.

[8] Arndt, M., Bigelow, B., "Evidence-based management in health care organizations: A cautionary note", Health care management review, 34(3), 206-13, 2009.

[9] Hewison, A., "Evidence-based medicine: what about evidence-based management?", Journal of Nursing Management, 5(4), 195-198, 1997.

[10] Janati, A., Hasanpoor, E., Hajebrahimi, S., Sadeghi-Bazargani, H., "Health care managers' perspectives on the sources of evidence in evidence-based hospital management: A qualitative study in Iran", Ethiopian journal of health sciences, 27(6), 659-68, 2017.

[11] Center for Evidence-Based Management. What is evidence-based management? 2015. Available at: http://www. cebma.org/\#what-is evidence-based-management.

[12] Barends, EG., Briner, RB., "Teaching evidence-based practice: lessons from the pioneers: an interview with Amanda Burls and Gordon Guyatt", Academy of Management Learning \& Education, 13(3), 476-83, 2014. 
[13] Guo R, Farnsworth TJ, Hermanson PM, "Information resources for hospital administrator health care management decision-making”, Journal of hospital librarianship, 3,15(3), 274-83, 2015.

[14] Bongers, I., "Reflective management practice: a matter of rigor and relevance", paper presented at European Health Management Annual Conference, Breda, 2015.

[15] HakemZadeh F, Baba VV., "Toward a theory of collaboration for evidence-based management", Management Decision, 54(10), 2587-616, 2016.

[16] Guo, R., Berkshire, SD., Fulton, LV., Hermanson, PM., "Use of evidence-based management in healthcare administration decision-making”, Leadership in Health Services, 30(3), 330-42, 2017.

[17] Rousseau, DM., "Is there such a thing as “evidence-based management?", Academy of management review, 31(2), 256-69, 2006.

[18] Walshe, K., Rundall, TG., "Evidence-based management: from theory to practice in health care", The Milbank Quarterly, 79(3), 429-57, 2001.

[19] Rousseau, DM., Manning, J., Denyer, D., "11 Evidence in management and organizational science: assembling the field's full weight of scientific knowledge through syntheses", The academy of management annals, 2(1), 475-515, 2008.

[20] Hewison, A., "Evidence-based management in the NHS: is it possible?", Journal of health organization and management, 18(5), 336-48, 2004.

[21] Axelsson, R., "Towards an evidence-based health care management", The International journal of health planning and management, 13(4), 307-17, 1998.

[22] Baba, V., "Toward a paradigm for management”, Metamorphosis, 3(2), 132-42, 2004.

[23] Goodman, SN., Royall, R., "Evidence and scientific research", American Journal of Public Health, 78(12), 1568-74, 1988.

[24] Sackett, DL., Rosenberg, WM., Gray, JM., Haynes, RB., Richardson WS, "Evidence-based medicine: what it is and what it isn't", British Medical Journal, 312, 71-72, 1996.

[25] Chen, YH., Liu, CF., Hwang, HG., "Key factors affecting healthcare professionals to adopt knowledge management: The case of infection control departments of Taiwanese hospitals", Expert Systems with Applications, 38(1), 450-7, 2011.

[26] Tolfo, C., Wazlawick, RS., "The influence of organizational culture on the adoption of extreme programming”, Journal of systems and software, 81(11), 1955-67, 2008.

[27] Wallach, EJ., "Individuals and organizations: The cultural match", Training \& Development Journal, 37(2), 28-36, 1983.

[28] Quinn, RE., Beyond rational management: Mastering the paradoxes and competing demands of high performance, Jossey-Bass, USA, 1988.

[29] Deshpandé, R., Farley, JU., "Executive insights: corporate culture and market orientation: comparing Indian and Japanese firms", Journal of International Marketing, 7(4), 111-27, 1999. 
[30] Eddama O, Coast J, "A systematic review of the use of economic evaluation in local decisionmaking”, Health Policy, 86(2-3), 129-141, 2008.

[31] Hyder, AA., Corluka, A., Winch, PJ., El-Shinnawy, A., Ghassany, H., Malekafzali, H., Lim, MK., Mfutso-Bengo, J., Segura, E., Ghaffar, A., "National policy-makers speak out: are researchers giving them what they need?", Health policy and planning, 26(1), 73-82, 2010.

[32] Greaves, DE., "Evidence-based management of Caribbean health systems: barriers and opportunities", International Journal of Health Governance, 22(2), 104-17, 2017.

[33] Reay, T., Berta, W., Kohn, MK., "What's the evidence on evidence-based management?", Academy of Management Perspectives, 23(4),5-18, 2009.

[34] Johns, G., "The essential impact of context on organizational behavior", Academy of management review, 31(2), 386-408, 2006.

[35] Adler, NJ., "A typology of management studies involving culture”, Journal of international business studies, 14(2), 29-47, 1983.

[36] Cappelli, P., "The missing role of context in OB: The need for a meso-level approach", Organizational Behavior, 13, 55-110, 1991.

[37] Rousseau, DM., Fried, Y., "Location: Contextualizing organizational research”, Journal of Organizational Behavior: The International Journal of Industrial, Occupational and Organizational Psychology and Behavior, 22(1), 1-3, 2001.

[38] Grimshaw, JM., Eccles, MP, Walker, AE., Thomas, RE., "Changing physicians' behavior: what works and thoughts on getting more things to work", J Contin Educ Health Prof, 22(4), 237-243, 2002 .

[39] Hawe, P., Shiell, A., Riley, T., "Complex interventions: how "out of control" can a randomized controlled trial be?", BMJ, 328, 1561-1563, 2004.

[40] Rubenstein, LV., Pugh, J., "Strategies for promoting organizational and practice change by advancing implementation research", Journal of General Internal Medicine, 21(2), 58, 2006.

[41] Barends, E., Villenueva, J., Briner, RB., ten Have, S., "Managers' attitudes and perceived barriers to evidence-based management an international survey", PLoS ONE,12 (10), e0184594, 2017.

[42] Liang, Z., Howard, PF., Leggat, SG., Murphy, G., “A framework to improve evidence-informed decision-making in health service management", Australian Health Review, 36(3), 284-9, 2012.

[43] Kovner, AR., Rundall, TG., "Evidence-based management reconsidered", Frontiers of health services management, 22(3), 3, 2006.

[44] Van de Ven, AH., Johnson, PE., "Knowledge for theory and practice", Academy of management review, 31(4), 802-21, 2006.

[45] Alexander, JA., Hearld, LR., Jiang, HJ., Fraser, I., "Increasing the relevance of research to health care managers: Hospital CEO imperatives for improving quality and lowering costs", Health care management review, 32(2), 150-9, 2007. 
[46] Rousseau, D. M., Evidence-based management in health care, in: C. Korunka \& P. Hoffmann (Eds.), Change and quality in human service work, Munich: Hampp, pp. 33-46, 2005.

[47] Rousseau DM, Gunia BC, "Evidence-based practice: The psychology of EBP implementation", Annual Review of Psychology, 67, 667-92, 2016.

[48] Lee, RP., Chen, Q., Hartmann, NN., "Enhancing stock market return with new product preannouncements: the role of information quality and innovativeness", Journal of Product Innovation Management, 33(4), 455-71, 2016.

[49] Bresnen, M., Hodgson, D., Bailey, S., Hyde, P., Hassard, J., "Being a manager, becoming a professional? A case study and interview-based exploration of the use of management knowledge across communities of practice in healthcare organizations", Health Services and Delivery Research, 2(14), 1-138, 2014.

[50] Zaltman, G., Moorman, C., "The importance of personal trust in the use of research". Journal of Advertising Research, 28(5), 16-24, 1988.

[51] Kitson, A., Harvey, G., McCormack, B., "Enabling the implementation of evidence-based practice: a conceptual framework", BMJ Quality \& Safety, 7(3), 149-58, 1998.

[52] Kulik, CT., "Working below and above the line: the research-practice gap in diversity management. Human Resource Management Journal, 24(2), 129-44, 2014.

[53] HakemZadeh, F., Baba, VV., "Measuring the actionability of evidence for evidence-based management", Decision, 54(5), 1183-204, 2016.

[54] Briner, RB., Denyer D., Rousseau, DM., "Evidence-based management: concept cleanup time?", Academy of Management Perspectives, 23(4), 19-32, 2009.

[55] Bordoloi, P., Islam, N., Knowledge management practices and healthcare delivery: a contingency framework. The Electronic Journal of Knowledge Management, 10(2), 110-20, 2012.

[56] Hongsermeier, T., Maviglia, S., Tsurikova, L., Bogaty, D., Rocha, RA., Goldberg, H., Meltzer, S., Middleton, B., "A legal framework to enable sharing of Clinical Decision Support knowledge and services across institutional boundaries”, In AMIA Annual Symposium Proceedings, pp. 925, 2011.

[57] Arntzen-Bechina, A., Leguy, C., "An insight into knowledge flow in biomedical engineering science", The Electronic Journal of Knowledge Management, 5(2), 153-60, 2007.

[58] Boateng, W., "Knowledge Management in Evidence-Based Medical Practice: Does the Patient Matter?", Electronic Journal of Knowledge Management, 8(3), 281, 2010.

[59] Turner, S., Quasi-science and the state, in Governing science in comparative perspective Governance of knowledge (Ed. S. Nico), Routledge, United Kingdom, pp. 255-284, 2017.

[60] Martelli, PF., Hayirli, TC., "Three perspectives on evidence-based management: rank, fit, variety", Management Decision”, 56(10), 2085-100, 2018. 
[61] Kothari, A., Hovanec, N., Hastie, R., Sibbald, S., "Lessons from the business sector for successful knowledge management in health care: a systematic review", BMC health services research, 11(1), 173, 2011.

[62] Von Krogh, G., Ichijo K., Nonaka, I., Enabling knowledge creation: How to unlock the mystery of tacit knowledge and release the power of innovation, Oxford University Press on Demand, 2000.

[63] Chung, YC., Hsu, Y-W., Peng, J T., Tsai, CH., Huang, HL., “A study of the correlation between knowledge management activities and operational performance in Taiwan hospital", Information Technology Journal, 12(6), 1502-1511, 2013.

[64] Green, LW., Ottoson, JM., Garcia, C., Hiatt, RA., "Diffusion theory and knowledge dissemination, utilization, and integration in public health", Annual review of public health, 30 , 151-74, 2009.

[65] Cunliffe, AL., Jun, JS., "The need for reflexivity in public administration", Administration \& Society, 37(2), 225-42, 2005.

[66] Krogh, GV., Roos, J., Slocum, K., “An essay on corporate epistemology”, Strategic management journal, 15(2), 53-71, 1994.

[67] Glazer R. "Measuring the knower: Towards a theory of knowledge equity", California management review, 40(3), 175-94, 1998.

[68] Orlikowski, WJ., "Knowing in practice: Enacting a collective capability in distributed organizing”, Organization Science, 13(3), 249-73, 2002.

[69] Von Hippel, E., "Sticky information" and the locus of problem-solving: implications for innovation", Management Science, 40(4), 429-39, 1994.

[70] Dobbins, M., DeCorby, K., Robeson, P., Husson, H., Tirilis, D., Greco, L., “A knowledge management tool for public health: health-evidence.ca”, BMC public health, 10, 496, 2010.

[71] Singh, MD., Kant, R., "Knowledge management barriers: An interpretive structural modeling approach", International Journal of Management Science and Engineering Management, 3(2), 141-50, 2008.

[72] Choy, CS., Suk, CY., "Critical factors in the successful implementation of knowledge management", Journal of Knowledge Management Practice, 6(1), 234-58, 2005.

[73] Liang, Z., Howard, P., "Evidence-informed managerial decision-making: What evidence counts?: (Part two)", Asia Pacific Journal of Health Management, 6(2), 12, 2011.

[74] Meyer, JW., ed. National development and the world system: educational, economic, and political change, University of Chicago Press, 1950-1970, 1979.

[75] McDaniel, Jr., Reuben, R., Jordan, ME., Fleeman, BF., "Surprise, surprise, surprise! A complexity science view of the unexpected", Health Care Management Review, 28(3), 266-78, 2003. 